\title{
FULL LUTZ TWIST ALONG THE BINDING OF AN OPEN BOOK
}

\author{
BURAK OZBAGCI AND MEHMETCIK PAMUK
}

\begin{abstract}
Let $T$ denote a binding component of an open book $(\Sigma, \phi)$ compatible with a closed contact 3-manifold $(M, \xi)$. We describe an explicit open book $\left(\Sigma^{\prime}, \phi^{\prime}\right)$ compatible with $(M, \zeta)$, where $\zeta$ is the contact structure obtained from $\xi$ by performing a full Lutz twist along $T$. Here, $\left(\Sigma^{\prime}, \phi^{\prime}\right)$ is obtained from $(\Sigma, \phi)$ by a local modification near the binding.
\end{abstract}

\section{INTRODUCTION}

Let $T$ denote a binding component of an open book $(\Sigma, \phi)$ compatible with a closed contact 3-manifold $(M, \xi)$. Then, by definition, $T$ is a transverse knot. By performing a full Lutz twist along $T$, we get a new contact structure $\zeta$ on $M$. Our intention in the present note is to give an explicit open book $\left(\Sigma^{\prime}, \phi^{\prime}\right)$ compatible with $(M, \zeta)$.

Our construction can be outlined as follows. First we use the fact that there is a Legendrian approximation $L_{1}$ of the binding component $T$, which is included in a page $\Sigma$ (see [20]). Then we express the effect of a full Lutz twist along $T$ by a contact $(+1)$-surgery on a four-component link $\mathbb{L}=L_{1} \sqcup L_{2} \sqcup L_{3} \sqcup L_{4}$ in $M$, where $L_{2}$ is a Legendrian push-off of $L_{1}$ with two additional up-zigzags, $L_{3}$ is a Legendrian push-off of $L_{2}$ and $L_{4}$ is a Legendrian push-off of $L_{3}$ with two additional up-zigzags. This result is, indeed, analogues to the results in [4, 9]. Next, we stabilize the open book at hand to embed all four components of the Legendrian link $\mathbb{L}$ into a page (cf. [11]). Finally, we use the fact that, a contact $(+1)$-surgery on $\mathbb{L}$ corresponds to additional lefthanded Dehn twists along each $L_{i}(i=1, \ldots, 4)$, on the page. As a result, we observe that $\left(\Sigma^{\prime}, \phi^{\prime}\right)$ is obtained from $(\Sigma, \phi)$ by a local modification near the binding and, by construction, the genus of $\Sigma$ is the same as the genus of $\Sigma^{\prime}$.

With an other point of view, what we describe in this note is a planar relative open book that is compatible with the contact $T^{2} \times I$ layer we "insert" while performing a full Lutz twist. Relative open books for contact 3-manifolds whose boundary consists of convex tori were introduced and studied by Van Horn-Morris in his thesis [15]. In particular, he describes a relative open book compatible with a basic slice. It turns out that the relative open book we obtain for the full Lutz twist through contact surgery, can be essentially obtained by appropriately gluing together a string of relative open books for the basic slice.

Throughout this paper, we assume that all contact structures are positive and co-oriented, all transverse knots are positively transverse, and all stabilizations are positive. The reader may turn to [10, 11, 13, 19] for the basic material on contact topology.

2000 Mathematics Subject Classification. 57R17, 57R65.

Key words and phrases. Lutz twist, contact surgery, open book decomposition. 


\section{LUTZ TWISTS}

Let $T$ be a knot transverse to the contact structure $\xi$ in a 3-manifold $M$. Then, in suitable local coordinates, we can identify $T$ with $S^{1} \times\{0\} \subset S^{1} \times D_{\delta}^{2}$ for some, possibly small $\delta>0$ such that $\xi=\operatorname{ker}\left(d \theta+r^{2} d \varphi\right)$ and $\partial_{\theta}$ corresponds to the positive orientation of $T$. In order to simplify the notation, we will work with $S^{1} \times D^{2}$ as a local model. A simple Lutz twist along $T$ is defined by replacing the contact structure $\xi$ on $M$ by $\xi^{T}$ which coincides with $\xi$ outside the solid torus $S^{1} \times D^{2}$ and on $S^{1} \times D^{2}$ is given by

$$
\operatorname{ker}\left(h_{1}(r) d \theta+h_{2}(r) d \varphi\right)
$$

where $h_{1}, h_{2}:[0,1] \rightarrow \mathbb{R}$ are smooth functions satisfying the following conditions:

(i) $h_{1}(r)=-1$ and $h_{2}(r)=-r^{2}$ near $r=0$,

(ii) $h_{1}(r)=1$ and $h_{2}(r)=r^{2}$ near $r=1$,

(iii) $\left(h_{1}(r), h_{2}(r)\right)$ is never parallel to $\left(h_{1}^{\prime}(r), h_{2}^{\prime}(r)\right)$ for $r \neq 0$.

Note that $\xi^{T}$ is well-defined up to isotopy, i.e., the isotopy class of $\xi^{T}$ does not depend on the particular choice of the functions $h_{1}$ and $h_{2}$. Moreover, it is clear that a simple Lutz twist does not change the topology of the underlying 3-manifold, but, in general, $\xi$ and $\xi^{T}$ are not homotopic as oriented 2-plane fields (see [13, Section 4.3]).

A full Lutz twist along $T$ is defined similar to a simple Lutz twist but the boundary conditions (i) and (ii) above are replaced by

$$
h_{1}(r)=1 \text { and } h_{2}(r)=r^{2} \text { for } r \in[0, \varepsilon] \cup[1-\varepsilon, 1]
$$

for some small $\varepsilon$, (iii) still holds and we require that the curve $\left(h_{1}(r), h_{2}(r)\right)$ completes a full turn around the origin. A full Lutz twist does not change the homotopy class of the contact structure as a 2-plane field, nor the topology of the underlying manifold (see [13, Proposition 4.5.4]). Let $\zeta$ denote the contact structure obtained by applying a full Lutz twist along $T$. Note that $\left(M, \xi^{T}\right)$ and $(M, \zeta)$ are both overtwisted.

Remark 2.1. Full Lutz twist is equivalent to replacing the contact structure in the $T^{2} \times I$ layer (corresponding to $r \in I=[\epsilon, 1-\epsilon]$ ) by another contact structure with an additional $2 \pi$-twist in the $I$-direction.

\section{THE SURGERY DIAGRAM FOR A FULL LUTZ TWIST}

In a recent series of papers [1, 2, 3], a notion of contact $r$-surgery along Legendrian knots in contact 3-manifolds is described, where $r \in(\mathbb{Q} \backslash\{0\}) \cup\{\infty\}$ denotes the framing relative to the natural contact framing. This generalizes the contact surgery introduced by Eliashberg [6] and Weinstein [21], which corresponds to the contact (-1)-surgery.

On the other hand, the classical notion of a Lutz twist (see [16, 17]) played an important role in constructing various contact structures. It turns out that, a simple Lutz twist along a transverse knot in a contact 3-manifold is equivalent to contact $(+1)$-surgery along a Legendrian two-component link [2]. Moreover, an explicit Legendrian surgery description for the simple Lutz twist is given in [4, 9]. Similarly, a full Lutz twist along a transverse knot in a contact 3 -manifold is equivalent to contact $(+1)$-surgery along a Legendrian four-component link (cf. [2, 12]). The idea here is that a full Lutz twist is equivalent to two iterated simple Lutz twists. 
Theorem 3.1. Let $L_{1}$ be an oriented Legendrian knot in $(M, \xi)$, represented by its front projection in $\left(\mathbb{R}^{3}, \xi_{\text {st }}\right)$ disjoint from the link describing $(M, \xi)$ and $L_{2}$ be a Legendrian push-off of $L_{1}$ with two additional up-zigzags, $L_{3}$ be a Legendrian push-off of $L_{2}$ and $L_{4}$ be a Legendrian push-off of $L_{3}$ with two additional up-zigzags. Let $\mathbb{L}:=L_{1} \sqcup L_{2} \sqcup L_{3} \sqcup L_{4}$ (see Figure 1) and $\xi^{\prime}$ be the contact structure obtained from $\xi$ by contact $(+1)$-surgery on $\mathbb{L}$. If $\zeta$ denotes the contact structure obtained from $\xi$ by a full Lutz twist along a positive transverse push-off $T$ of $L_{1}$, then $\xi^{\prime}$ and $\zeta$ are isotopic.

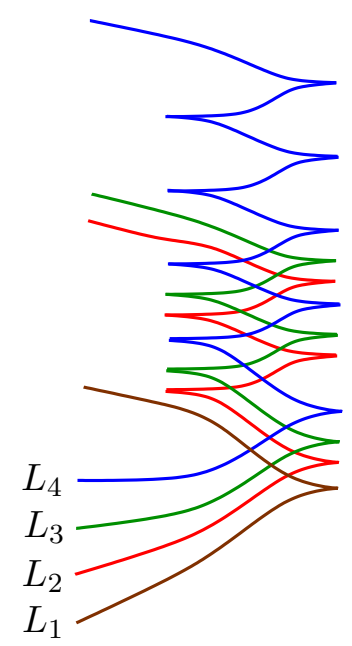

FIGURE 1. Legendrian link $\mathbb{L}=L_{1} \sqcup L_{2} \sqcup L_{3} \sqcup L_{4}$

Proof. We first show that contact $(+1)$-surgery on the Legendrian link $\mathbb{L}$ does not topologically change the underlying manifold $M$. To see this, note that an additional zigzag adds a negative twist to the contact framing. Hence, topologically contact $(+1)$-surgery on $L_{4}$ is the same as a contact $(-1)$-surgery along a Legendrian push-off of $L_{3}$. Therefore, by [1, Proposition 8], the contact $(+1)$-surgery on $L_{3}$ topologically cancels out the contact $(+1)$-surgery on $L_{4}$. The same argument holds for the contact $(+1)$-surgeries on $L_{1}$ and $L_{2}$.

We know that $\zeta$ is overtwisted. It is not too hard to see that $\xi^{\prime}$ is also overtwisted (cf. [18]). Once we show that $\xi^{\prime}$ is homotopic to $\zeta$ as an oriented 2-plane field, then the result immediately follows from Eliashberg's classification of overtwisted contact structures [5]. Since a full Lutz twist does not change the homotopy class of $\xi$ as a 2-plane field, i.e., $\xi$ is homotopic to $\zeta$, we need to verify that $\xi$ is homotopic to $\xi^{\prime}$. Recall that for any two 2-plane fields $\xi$ and $\xi^{\prime}$ on $M$, there is an obstruction $d^{2}\left(\xi, \xi^{\prime}\right) \in H^{2}(M ; \mathbb{Z})$ for $\xi$ to be homotopic to $\xi^{\prime}$ over the 2-skeleton of $M$ and if $d^{2}\left(\xi, \xi^{\prime}\right)=0$, after applying a homotopy which takes $\xi$ to $\xi^{\prime}$ over the 2-skeleton, there is another obstruction $d^{3}\left(\xi, \xi^{\prime}\right)$ for $\xi$ to be homotopic to $\xi^{\prime}$ over all of $M$.

Consider the standard tight contact $\left(S^{1} \times S^{2}, \xi\right)$, which can be represented by contact $(+1)$ surgery on a Legendrian unknot $L_{0}$ with only two cusps. Let $L_{1}$ be a Legendrian push-off of $L_{0}$. Note that, by the neighborhood theorem for Legendrian knots, it suffices to prove the vanishing of the two-dimensional obstruction $d^{2}\left(\xi, \xi^{\prime}\right)$ for this particular $L_{1}$ (cf. [4]). It is well-known that 
$e(\xi)=0$. Here we claim that $e\left(\xi^{\prime}\right)=0$, as well. It follows that $d^{2}\left(\xi, \xi^{\prime}\right)=0$, by the formula $2 d^{2}\left(\xi, \xi^{\prime}\right)=e(\xi)-e\left(\xi^{\prime}\right)$ (see [13, Remark 4.3.4]).

The Thurston-Bennequin invariants of the Legendrian knots $L_{0}, L_{1}, \ldots, L_{4}$ can easily be computed from their front projections as $t b\left(L_{0}\right)=-1, t b\left(L_{1}\right)=-1, t b\left(L_{2}\right)=t b\left(L_{3}\right)=-3$ and $t b\left(L_{4}\right)=-5$. Thus, the topological framings of the surgeries are given by $\operatorname{tf}\left(L_{0}\right)=$ $t f\left(L_{1}\right)=0, t f\left(L_{2}\right)=t f\left(L_{3}\right)=-2$ and $t f\left(L_{4}\right)=-4$. Write $\mu_{i}$ for the meridional circle to $L_{i}$ as well as the homology classes they represent in the homology of the surgered manifold. It is well-known that $H_{1}(M ; \mathbb{Z})$ is generated by the meridians $\left\{\mu_{0}, \ldots, \mu_{4}\right\}$ with relations given by

$$
t f\left(L_{i}\right) \mu_{i}+\sum_{j \neq i} l k\left(L_{i}, L_{j}\right) \mu_{j}=0, i=0, \ldots, 4 .
$$

These equations imply that $\mu_{0}=\mu_{1}=\mu_{4}=-\mu_{2}=-\mu_{3}$. Now with PD denoting the Poincaré duality isomorphism, we have (see [3, Corollary 3.6])

$$
\begin{aligned}
e\left(\xi^{\prime}\right) & =\Sigma_{i=1}^{4} \operatorname{rot}\left(L_{i}\right) \mathrm{PD}^{-1}\left(\mu_{i}\right) \\
& =-2 \mathrm{PD}^{-1}\left(\mu_{2}\right)-2 \mathrm{PD}^{-1}\left(\mu_{3}\right)-4 \mathrm{PD}^{-1}\left(\mu_{4}\right)=0 .
\end{aligned}
$$

Finally, let us see the effect of the surgery along $\mathbb{L}$ on the 3 -dimensional obstruction. It is sufficient to consider an oriented knot $L_{1}$ in $\left(S^{3}, \xi_{s t}\right)$. The absolute $d_{3}$-invariant (for 2-plane fields in $S^{3}$ ) of the contact structure $\xi^{\prime}$ obtained by these surgeries is given by (see [3, Corollary 3.6])

$$
d_{3}\left(\xi^{\prime}\right)=\frac{1}{4}\left(c^{2}-3 \sigma(X)-2 \chi(X)\right)+q
$$

where $X$ denotes the handlebody obtained from $D^{4}$ by attaching four 2-handles corresponding to the surgeries, $q$ denotes the number of components in $\mathbb{L}$ on which we perform $(+1)$ surgery and $c \in H^{2}(X ; \mathbb{Z})$ is given by $c\left(\left[\Sigma_{i}\right]\right)=\operatorname{rot}\left(L_{i}\right)$ on $\left[\Sigma_{i}\right] \in H_{2}(X ; \mathbb{Z})$ where $\Sigma_{i}$ is the Seifert surface for $L_{i}$. It is clear that $\chi(X)=5$.

Lemma 3.2. We have $\sigma(X)=0$ and $c^{2}=-8$.

Proof. Let $t$ denote the Thurston-Bennequin invariant of $L_{1}$. Hence we have $t b\left(L_{2}\right)=t b\left(L_{3}\right)=$ $t-2$ and $t b\left(L_{4}\right)=t-4$. Then the topological framings of the surgeries are

$$
t f\left(L_{1}\right)=t+1, t f\left(L_{2}\right)=t f\left(L_{3}\right)=t-1, \text { and } t f\left(L_{4}\right)=t-3 .
$$

The linking number between $L_{1}$ and $L_{j}$ is given by $l k\left(L_{1}, L_{j}\right)=t$ for $j=2,3$ and 4 . Also we have $l k\left(L_{2}, L_{3}\right)=l k\left(L_{2}, L_{4}\right)=t-2$ and $l k\left(L_{3}, L_{4}\right)=t-2$. Then $\sigma(X)$ is the signature of the linking matrix

$$
\left[\begin{array}{cccc}
t+1 & t & t & t \\
t & t-1 & t-2 & t-2 \\
t & t-2 & t-1 & t-2 \\
t & t-2 & t-2 & t-3
\end{array}\right]
$$


If we slide $L_{4}$ over $L_{3}$ and slide $L_{2}$ and $L_{3}$ over $L_{1}$, then the linking matrix becomes

$$
A=\left[\begin{array}{cccc}
t+1 & -1 & -1 & 0 \\
-1 & 0 & -1 & 0 \\
-1 & -1 & 0 & -1 \\
0 & 0 & -1 & 0
\end{array}\right]
$$

The characteristic polynomial for the matrix $A$ is $\lambda^{4}-(t+1) \lambda^{3}-4 \lambda^{2}+2(t+2) \lambda+1$. By analyzing the coefficients of this polynomial one can see that the eigenvalues $\lambda_{1}, \ldots, \lambda_{4}$ satisfy the following equalities:

(i) $\lambda_{1} \lambda_{2}+\left(\lambda_{1}+\lambda_{2}\right)\left(\lambda_{3}+\lambda_{4}\right)+\lambda_{3} \lambda_{4}=-4$,

(ii) $\lambda_{1} \lambda_{2} \lambda_{3} \lambda_{4}=1$.

$A$ is a real symmetric matrix, so the eigenvalues must be real and by (ii) we have three cases for the eigenvalues of $A$ :

(I) all the eigenvalues are positive,

(II) all the eigenvalues are negative,

(III) there are two positive and two negative eigenvalues.

Clearly, (i) implies that the matrix $A$ has two positive and two negative eigenvalues and hence $\sigma(X)=0$.

In order to compute $c^{2}$, set $r=\operatorname{rot}\left(L_{1}\right)$. Then $\operatorname{rot}\left(L_{2}\right)=\operatorname{rot}\left(L_{3}\right)=r-2, \quad$ and $\operatorname{rot}\left(L_{4}\right)=$ $r-4$. As in Section 3 of [3], we have

$$
c^{2}=x r+y(r-2)+z(r-2)+w(r-4),
$$

where $(x, y, z, w)$ is the solution of the system of equations

$$
\left[\begin{array}{cccc}
t+1 & t & t & t \\
t & t-1 & t-2 & t-2 \\
t & t-2 & t-1 & t-2 \\
t & t-2 & t-2 & t-3
\end{array}\right]\left[\begin{array}{c}
x \\
y \\
z \\
w
\end{array}\right]=\left[\begin{array}{c}
r \\
r-2 \\
r-2 \\
r-4
\end{array}\right] .
$$

It follows that $x=r, y=z=-2-r, w=4+r$, and hence $c^{2}=-8$.

Consequently,

$$
d_{3}\left(\xi^{\prime}\right)=\frac{1}{4}\left(c^{2}-3 \sigma(X)-2 \chi(X)\right)+q=-1 / 2=d_{3}\left(\xi_{s t}\right)
$$

which implies that $d^{3}\left(\xi, \xi^{\prime}\right)=0$. Therefore, since $d^{2}\left(\xi, \xi^{\prime}\right)=d^{3}\left(\xi, \xi^{\prime}\right)=0$, we conclude that $\xi$ is homotopic to $\xi^{\prime}$, i.e., the contact $(+1)$-surgery on $\mathbb{L}$ does not change the homotopy class of the contact structure.

\section{THE EFFECT OF A FULl LUTZ TWIST ALONG THE BINDING OF AN OPEN BOOK}

In this section, we describe the effect of a full Lutz twist along the binding of an open book. Let $T$ denote a binding component of an open book $(\Sigma, \phi)$ compatible with a closed contact 3manifold $(M, \xi)$. The first step of our construction is to realize a push-off of $T$ as a Legendrian curve on the page $\Sigma$. If $T$ is not the only binding component of $(\Sigma, \phi)$, then a push-off of $T$ 
can be Legendrian realized on $\Sigma$, otherwise this is impossible. The next result, which is due to Vela-Vick [20], allows us to handle the case where the binding of $(\Sigma, \phi)$ is connected.

Lemma 4.1. Suppose that $T$ is the only binding component of $(\Sigma, \phi)$. Then there is a Legendrian approximation $L_{1}$ of $T$, realized as a curve on a page of the open book $\left(\Sigma_{1}, \phi_{1}\right)$ obtained from $(\Sigma, \phi)$ by stabilizing once, without changing the transverse isotopy type of $T$.

Proof. Although this was shown in [20, Lemma 3.1], here we give an alternative proof of this fact. Suppose that $T$ is the only binding component of $(\Sigma, \phi)$. We take a small meridional circle $m$ linking $T$ once and hence intersecting every page $\Sigma$ transversely. Now we perform +1 surgery along $m$ and extend the open book into the glued in solid torus as follows (see [8]): Foliate the solid torus we glue in by annuli so that each annulus has the core of this solid torus as one of its boundary components. The other boundary component of each annulus is a $(1,1)$ curve which is identified with a meridional circle to $m$ during the surgery. So we delete a disk from every page $\Sigma$ and glue in an annulus to get an open book with page $\Sigma_{1}$ and monodromy $\phi_{1}$, where $\phi_{1}$ is obtained from $\phi$ by composing with a right-handed Dehn twist along a curve parallel to the newly introduced binding component. In fact, we just stabilized $(\Sigma, \phi)$. The contact structure compatible with $\left(\Sigma_{1}, \phi_{1}\right)$ is isotopic to $\xi$ and we did not touch the transverse knot $T$ in $M$ while performing the surgery.

$T$

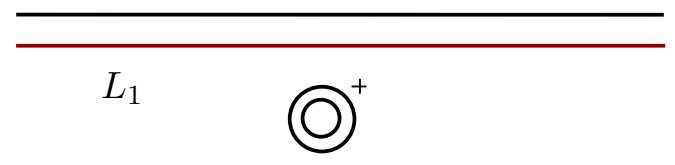

FIGURE 2. Legendrian knot $L_{1}$ on the page $\Sigma_{1}$

The knot $L_{1}$ which is a push-off of $T$ on the new page $\Sigma_{1}$ as shown abstractly in Figure 2 can now be Legendrian realized since it is homologically nontrivial on $\Sigma_{1}$. Consequently, $L_{1}$ is a Legendrian knot on the page $\Sigma_{1}$ whose positive transverse push-off is $T$.

Since $L_{2}$ is obtained from a push-off of $L_{1}$ by adding two zigzags, we can realize $L_{2}$ on a page of an open book $\left(\Sigma_{2}, \phi_{2}\right)$ obtained by stabilizing $\left(\Sigma_{1}, \phi_{1}\right)$ twice (cf. [9]). To be more precise, $L_{2}$ is a push-off of $L_{1}$ on $\Sigma_{2}$, except that $L_{2}$ goes over the two new 1-handles glued to $\Sigma_{1}$ in the stabilization process. By continuing in this manner, we see that there is an open book $(\Sigma, \phi)$, compatible with $(M, \xi)$, containing the Legendrian link $\mathbb{L}$ on a page. Then the open book $\left(\Sigma, \phi \circ D_{\mathbb{L}}^{-}\right)$is compatible with $\left(M, \xi^{\prime}\right)$, where $D_{\mathbb{L}}^{-}$denote the composition of left-handed Dehn twists along each component of the link $\mathbb{L} \subset \Sigma$ (see Figure 3). Consequently, by Giroux's correspondence [14] coupled with Theorem 3.1, we conclude that $\left(\Sigma, \phi \circ D_{\mathbb{L}}^{-}\right)$is compatible with $(M, \zeta)$.

Remark 4.2. The discussion above gives an explicit relative open book for the full Lutz twist. In other words, the contact $T^{2} \times I$ layer with a $2 \pi$-twist inserted while performing a full Lutz twist has a compatible relative open book whose page is a six-punctured sphere, and whose 


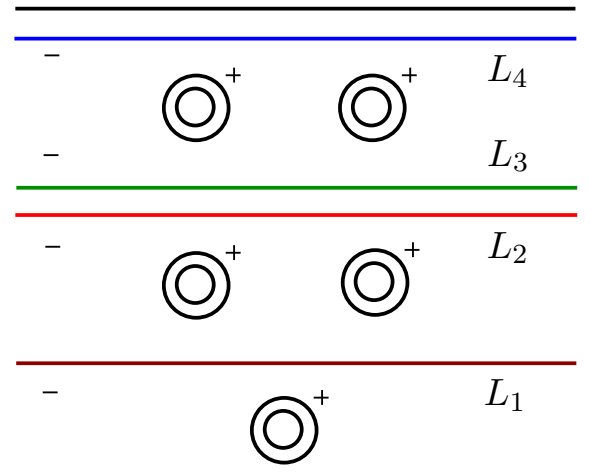

FIGURE 3. Modification near the binding which corresponds to the effect of a full Lutz twist. The solid curves without a sign are the binding components.

monodromy is the product of the Dehn twists along the curves shown in Figure 4 . Note that a relative open book compatible with a basic slice is described in Section 4.2 in [15]. It turns out that the relative open book we obtain for the full Lutz twist through contact surgery, can be essentially obtained by appropriately gluing together a string of relative open books compatible with the basic slice.

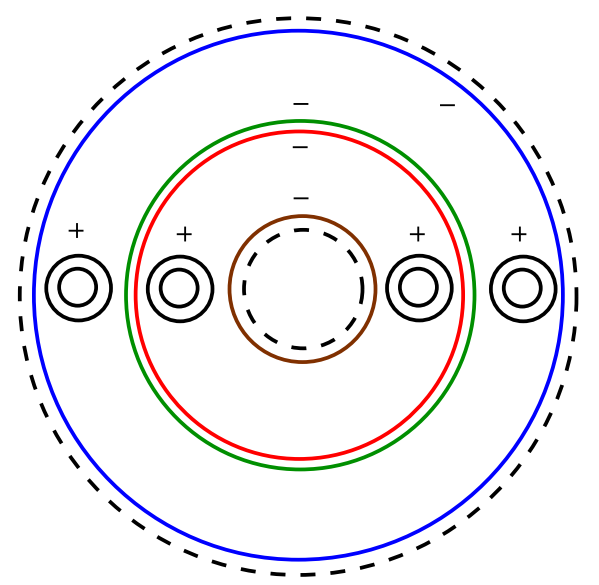

FIGURE 4. Relative open book compatible with the contact $T^{2} \times I$ layer with a $2 \pi$-twist in the $I$-direction. The dashed curves induce the boundary $\partial\left(T^{2} \times I\right)$.

Acknowledgements. We are grateful to Hansjörg Geiges and John B. Etnyre for helpful conversations. The authors were partially supported by the research grant $107 \mathrm{~T} 053$ of the Scientific and Technological Research Council of Turkey.

\section{REFERENCES}

1. F. Ding and H. Geiges, Symplectic fillability of tight contact structures on torus bundles, Algebr. Geom. Topol. 1 (2001), 153-172. 
2. F. Ding and H. Geiges, A Legendrian surgery presentation of contact 3-manifolds, Math. Proc. Cambridge Philos. Soc. 136 (2004), 583-598.

3. F. Ding, H. Geiges and A. I. Stipsicz, Surgery Diagrams for contact 3-manifolds, Turkish J. Math. 28 (2004), 41-74 (Proceedindgs of the 10th Gökova Geometry-Topology Conference 2003, 41-74)

4. F. Ding, H. Geiges and A. I. Stipsicz, Lutz twist and contact surgery, Asian J. Math. 9 (2005) 57-64

5. Y. Eliashberg, Classification of overtwisted contact structures on 3-manifolds, Invent. Math. 98 (1989), no. 3, 623-637.

6. Y. Eliashberg, Topological characterization of Stein manifolds of dimension $>2$, Internat. J. Math. 1 (1990), no. 1, 29-46.

7. J. Epstein, D. Fuchs, and M. Meyer, Chekanov-Eliashberg invariants and transverse approximations of Legendrian knots, Pacific J. Math. 201 (2001), no. 1, 89-106.

8. T. Etgü and B. Ozbagci, Explicit horizontal open books on some plumbings, Internat. J. Math. 17 (2006), no. 9, 1013-1031.

9. J. B. Etnyre, Planar open book decompositions and contact structures, Int. Math. Res. Not. 2004, no. 79, $4255-4267$.

10. J. B. Etnyre, Legendrian and transversal knots, Handbook of knot theory, 105-185, Elsevier B. V., Amsterdam, 2005.

11. J. B. Etnyre, Lectures on Open book Decompositions and Contact Structures, Floer Homology, Gauge Theory, and Low-Dimensional Topology, Clay Math. Proc. 20, Amer. Math. Soc., Providence (2006), 103-141.

12. J. B. Etnyre and K. Honda, On symplectic cobordisms, Math. Ann. 323 (2002), 31-39.

13. H. Geiges, An Introduction to Contact Topology, Cambridge studies in advanced mathematics 109, Cambridge University Press (2008).

14. E. Giroux, Contact Geometry: from dimension three to higher dimensions, Proceedings of the International Congress of Mathematicians (Beijing 2002), 405-414

15. J. Van Horn-Morris, Construction of open book decompositions, Ph.D. Thesis, UT Austin, 2007.

16. R. Lutz, Sur l'existence de certaines formes différentielles remarquables sur la sphére $S^{3}, \mathrm{C}$. R. Acad. Sci. Paris Sér. A-B 270 (1970), A1597-A1599.

17. J. Martinet, Formes de contact sur les variétés de dimension 3, Proceedings of the Liverpool Singularities Symposium II, Lecture Notes in Math. 209, Springer-Verlag, Berlin, 1971, 142-163.

18. B. Ozbagci, A note on contact surgery diagrams, Internat. J. Math. 16 (2005), no. 1, 87-99.

19. B. Ozbagci and A. I. Stipsicz, Surgery on contact 3-manifolds and Stein surfaces, Bolyai Society Mathematical Studies, 13. Springer-Verlag, Berlin; János Bolyai Mathematical Society, Budapest, 2004.

20. D.S. Vela-Vick, On the transverse invariant for bindings of open books, arXiv:0806.1729.

21. A. Weinstein, Contact surgery and symplectic handlebodies, Hokkaido Math. J. 20 (1991), no. 2, 241-251.

Department of Mathematics, Koç University, Istanbul, TURKey

E-mail address: bozbagci@ku.edu.tr

E-mail address: mpamuk@ku.edu.tr 